\title{
Case Study:
}

\section{3 mix technique for Root canal failure treatment. *Dr. Ibrahim Khalil ${ }^{1}$, Dr. Shamim Ara Begum² , Dr. Md Amirul Islam ${ }^{3}$, Dr. Md Miftaul Islam $^{4}$, Dr. Mohammad Ali Kawsar ${ }^{5}$}

1. Associate Professor (CC) Head of Dental Unit, TMSS Medical College, Bogra 2. MBBS, MS Obs \& Gynae) MO, Medicine. Dhaka Dental College. 3. Assistant Professor, TMSS Medical College, Bogra 4. Associate Professor, TMSS Medical College-Dental Unit, Bogra. 5. Assistant Professor \& Head of Orthodontics Dept. TMSS Medical CollegeDental Unit, Bogra.

Article info: Received: March 22, 2016, Accepted: May 29, 2016,

\section{Abstract}

Progress has been made in understanding the nature of root canal infection and periapical disease. Sterilization of root canal system is one of the prominent problems. Evidences suggested that Enterococcus faecalis(E.faecalis) caused substantial root canal infections. So, elimination of such organismis important to achieve treatment success. Advance techniques and materials increase the success in Endodontics against Enterococcus faecalis. $3 \mathrm{mix}$ antibiotic therapy a new biologic approach in the treatment of carious lesions.

\section{Keywords:}

Enterococcus faecalis, Root canal infection, Periapical disease, 3 mix antibiotic

\section{Introduction:}

Since two decades evidence shows using of mixture of two or three antibiotic or with steroids to treating root canal infection. Shapiro LE et al and Roche Y et al (1997) used doxycicline, minocycline and metronidazole separately to evaluate the efficacy against Root Canal bacteria. Molander A, Dahlen G (2003) Evaluation of the antibacterial potential of Tetracycline or Erythromycin mixed with Calcium hydroxide as intracanal dressing against E. faecalis in vivo ${ }^{1}$. Z Mohammadi et al (February 2009) in a study entitled"Antibiotics as Intra canal Medicaments:

\section{Address of correspondence:}

Dr Ibrahim Khalil , BDS, MS

Associate Professor . TMSS Medical College Dental Unit. Bogra. E-mail: ibrahimps@yahoo.com Cell: 01911386083
A Review" here the author shown thatAntibiotics Tetracyclines have also been used as part of irrigating solutions and Clindamycin with a combination of three antibiotics (Metronidazole, Ciprofloxacin and Minocycline) have also been reported to be effective at reducing bacterial numbers in the root canal systems of infected teeth ${ }^{2}$. In 3 mix antibiotic or LSTR technique involves the use of three broad spectrum antibiotics namely- Metronidazole, Ciprofloxacin, and Minocycline which is developed by Professor Hoshino of the Niigata University School of Dentistry in 1988. is endorsing a 1:1:1 mix. The 3 antibiotics are mixed with Propylene Glycol or Macrogol, "which is found to be an excellent vehicle to carry $3 \mathrm{Mix}$ into the entire dentin (Image-A) and through the dentinal tubules and kill all the bacteria in lesions." According to Hoshino, $94 \%$ of the cases that experienced pain were relieved within 24 hours after use of the 
3Mix therapy. ${ }^{3}$ Mix antibiotic therapy is said to be "bactericidal to aerobic bacteria and to resistant obligate anaerobes." Hoshino, et. al. identify the anaerobic microorganisms recovered from carious dentin: $80 \%$ are obligate anaerobes from infected pulp; $92 \%$ are obligate anaerobes from infected root canal dentin; and $80 \%$ are obligate anaerobes. ${ }^{3,4,5}$ Obligate anaerobes are sensitive to Metronidazole which has wide bactericidal spectrum against anaerobes, the reason for its being first choice antibiotic. However, since not all bacteria like Enterococcus faecalis can be eliminated by Metronidazole alone, other drugs may be necessary to sterilize the infected dentin.

They have added Ciprofloxacin and Minocycline. The contention behind 3Mix is that "it sterilizes the lesion of an infected and softened dentin intentionally left during cavity preparation and the pulp." "3 Mix can eliminate bacteria from infected dental tissues of both deciduous and permanent teeth." 5, 6, 7, 8,9 . The assumption is that if eliminate bacteria, then eliminate the infection, thereby eliminating inflammation, and consequently, eliminating pain - both the acute and chronic type. According to Hoshino,"94\% of the cases that experienced pain were relieved within 24 hours after use of the 3Mix, regardless of the nature and severity of the pain." $6,7,8$
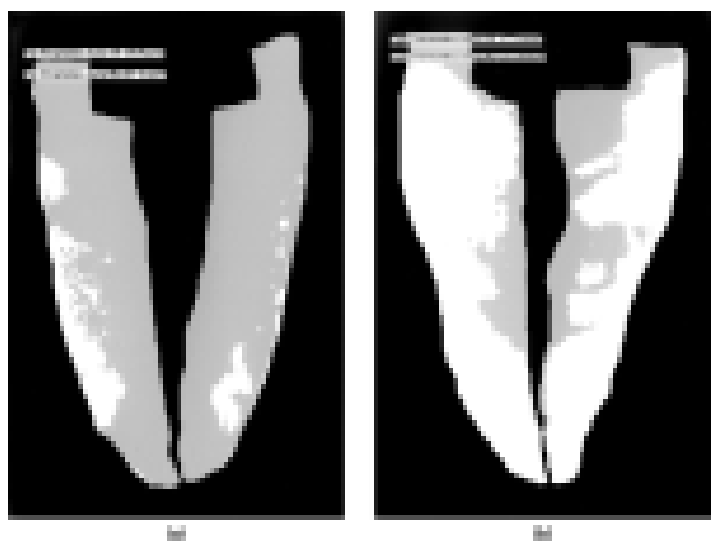

Image analysis of specimen showing percentage of surface area penetrated by dye (indicated by dark gray areas).These were used as vehicles: (a) Propylene glycol (b) Distilled water

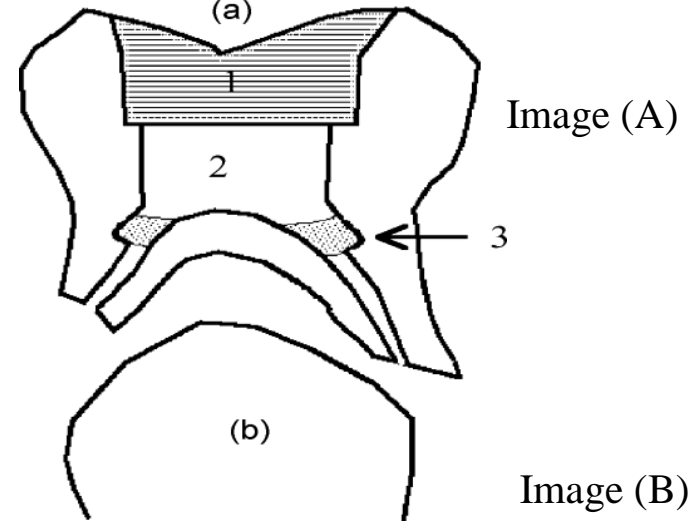

3) 3-mix antibiotic

2) Glass ionomer Fuji-ix

1) Inlay by resin cement

Image A \& B Courtesy -

Hoshino E. et. al Cariology research unit Niigata University School of Dentistry.

\section{Case Report:}

A 52 year-old male patient Named $\mathrm{Mr}$ Azizul Islam Bhuiyan was referred to TMSS Medical College Dental Unit for endodontic treatment of the right mandibular 2nd premolar with swelling and pain. Intra-oral clinical examination revealed that 2nd premolar had a carious lesion on the distal side and the right mandibular 1st molar had pocelain crown. The buccal mucosa presented red colored swelling. The patient denied spontaneous pain but reported painful situation upon percussion.

After endodontic access cavity preparation, cleaning with $0.9 \%$ Nacl sol. and dried. Here one thing should be considered that the cavity margin should be debris free and smear layer should be clean by using $15 \%$ E.D.T.A (Ethyl-di-methyl tetra acetic acid) or 5\% Na Hypo chloride solution in water, then placing the 3-Mix antibiotic on orifice of the root canal with the help of fine tips excavator then sealed with Glass Ionomer Fuji- ix GC and further reinforced with miracle mix GC.

The radiographic examination showed \{mage-C(1)\} the presence of external periapical resorption of whole root length of distal proximal area and a radiolucent lesion adjacent to the apex of tooth. 

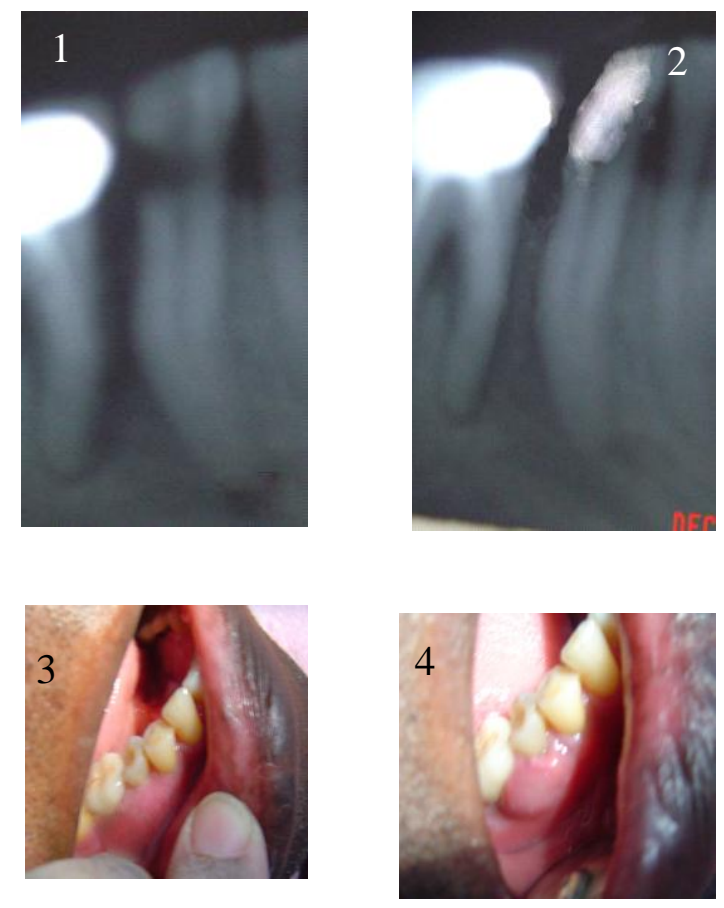

Image showing mild opacity (2) which indicate bone formation Between 2nd premolar and 1st molar.

Follow up had been taken after 24 hours the swelling \& pain dramatically relieved \{Image-C(4)\} and after 14 months the radiography revealed that new bone formation around the periapical region. $\{$ Image-C(2)\}

\section{Discussion:}

Enterococcus faecalis are more resistant bacteria. Therefore any presence of these bacteria may result Root Canal Treatment (RCT) failure. To save the tooth it is very important to eliminate these bacteria for a successful feedback. But resistant Enterococcus faecalis may impregnate in tiny dentinal tubules. It usually not killed by most of medicament or any single antibiotic. Several studies done using LSTR found excellent clinical results. The bactericidal effect of the LSTR 3Mix sterilization of the lesion . LSTR 3Mix MP therapy could kill all bacteria taken from carious lesions, necrotic pulps, infected root dentine and endodontic lesions of permanent teeth ${ }^{1,5,6}$
Endodontic management of teeth with severe pulpal necrosis with non vital pulp tissue, fistula, and loss of alveolar bone is generally discouraged. Sterilization of the root canal and periradicular region results in good healing of the periradicular region. Bacteria which are present mainly in the root canals and superficial layer of infected root canal wall may be easily removed by conventional root canal treatment. But the bacteria, which remain in the deep layers of root canal dentin, may leak out to periapical region and cause complications. Application of antibacterial drugs to endodontic lesions is one of the clinical procedures that can be used to sterilize such lesions. ${ }^{5,6,7}$. To sterilize such lesions, a single antibacterial drug may not be effective, even if it is a broad spectrum antibiotic. Reason being the bacterial composition of the infected root canals is a complex of resistant anaerobes and narrower dentinal tubules. In addition, bacteria may also invade root canals from other oral sites, e.g. dental plaque, saliva and from carious dentin which may also smear the root canal during endodontic treatment. All such bacteria should be targeted by antibacterial drugs. $5,6,7$

Since the overwhelming majority of bacteria in the deep layers of infected dentine of the root canal wall consist of obligate anaerobes, Metronidazole was selected as first choice among the antibacterial drugs. Metronidazole even at high concentrations cannot kill all the bacteria indicating the necessity of other drugs. Thus, Ciprofloxacin and Minocycline, in addition to Metronidazole were added to sterilize infected root dentin. ${ }^{3,5,6,7}$. To sterilize deep layers of infected root dentin, root canal medicaments should penetrate root canal dentin. The penetration ability of these drugs was improved by proper mixing of these drugs with Propylene Glycol and Macrogol to form ointment base. The penetration ability of Propylene Glycol and Macrogol ointment base was clearly demonstrated by Cruz et $a l^{3,8}$ 


\section{Conclusion}

Antibacterial mix (a combination of Ciprofloxacin, Metronidazole, and Minocycline mixed with Propylene Glycol and Macrogol) could be effective in root canal sterilization and in the RCT failure case by eliminating $E$. faecalis.

\section{References:}

1. Molander A, Dahlen G (2003) Evaluation of the antibacterial potential of tetracycline or erythromycin mixed withcalcium hydroxide as intracanal dressing against E. faecalis in vivo. Oral Surgery Oral Medicine Oral Pathology Oral Radiology Endodontics 96, 744-50.

2. Mohammadi Z, Farhad A, Ardakani FE (2009) "Antibiotics as Intra canal Medicaments: A Review". DentalResearch Journal 4, 48-52.

3. Shapiro LE, Knowles SR, Shear NH. Comparative safety of tetracycline, minocycline, and doxycycline. Arch Dermatol 1997;133:1224-1230.

4. Roche Y, Yoshimori RN. In vitro activity of spiramycin and metronidazoale alone and in combination against clinical isolates from odontogenic abscesses. Journal of Antimicorbial Chemotheraphy 1997;40:353.

5. Hoshino E, Takusige T. LSTR 3Mix-MP method, Better and LSTR therapy Dental Review 1998;666:57106.

6. Asgor MA, Ahmed M, Khandker NH, et al. LSTR 3MIX MP Therapy- a revolution in endodontic treatment: introduced in Bangladesh. Bangladesh Dental Journal 2005;17:5-9.

7. Garcia C, Cruz E, Hoshino E, et al. 3Mix Lesion Sterilization and Tissue Repair (LSTR) therapy in extensive caries of deciduous teeth. The J Philippine Dental Assoc 2004;56:4-13.

8. Souza V, Bernabe PFE, Holland R, et al. Periapical cyst repair after nonsurgical endodontic therapy: case report 1989;46:39-46.

9 .Aryanti $\mathrm{R}$, Hilbrand $\mathrm{H}$ et al, Determinants of Antimicrobial Use in the Developing World, Child Health Research Project Special Report, 2007;4:4-8 